\title{
JET PENETRATION IN GLASS
}

\author{
B. MORAN, L.A. GLENN and A. KUSUBOV \\ Lawrence Livermore National Laboratory, Livermore, CA \\ 94551-0808, U.S.A.
}

\begin{abstract}
We describe a phenomenological model which accounts for the mechanical response of glass to intense impulsive loading. An important aspect of this response is the dilatancy accompanying fracture. We have also conducted a number of experiments with $38.1-\mathrm{mm}$ diameter precision shaped charges to establish the performance against various targets and to allow evaluation of our model. At 3 charge diameters standoff, the data indicate that both virgin and damaged glass offer better (Bernoulli-scaled) resistance to penetration than either of 4340 steel, or 6061-T6 aluminum alloy. Timeresolved measurements indicate two distinct phases of jet penetration in glass: An initial hydrodynamic phase, and a second phase characterized by a slower penetration velocity. Our calculations show that at early time, a crater is formed around the jet and only the tip of the undisturbed jet interacts with the glass. At late time the glass has collapsed on the jet and degraded penetration continues via a disturbed and fragmented jet.
\end{abstract}

\section{Introduction.}

It is well known that jets fired against glass targets appeared to be bombarded by tiny fragments that destroyed alignment and reduced residual penetration [1]. More recently, X-Ray observations confirmed that, after penetrating a steel-glass-steel target, a jet became unaligned and fragmented [2]. A phenomenological model which accounts for the mechanical response of glass to intense loading has been proposed by Glenn et al., 1990 [3]. An important aspect of the model is the dilatancy accompanying the fracture. Dynamic experiments have shown that glass can bulk as much as $20 \%$ or more under impact loading [4]. In what follows we describe our phenomenological model and our jet penetration experiments. We also suggest further experiments and hydrocode simulations which would improve the predictive capability of jet penetration. This paper summarizes the main results in Moran et al [5] and Glenn et al [3].

\section{Constitutive Model.}

Our constitutive model for glass is described in Glenn et al [3]. The key point is illustrated in Figure 1 which shows pressure as a function of specific volume. Shock loading occurs along the Rayleigh line, after which unloading initiates along the adiabat (here shown coincident with the Hugoniot curve). At the onset of damage, the bulk modulus decreases and continued unloading departs from the adiabat. When the glass has unloaded to the initial specific volume, there is a residual compressive stress in the material. Computer calculations of jet penetration into glass show that this residual stress increases the inward radial acceleration of glass particles which then interact with the incoming jet. The model consists of three components: A scalar component of the stress tensor, a deviatoric stress tensor component, and most important a description of tensile failure. The initiation criterion which we used is based on limiting the most tensile principal stress component and can be extended to include the general stress-strain state. Upon initiation, a scalar damage parameter is assumed to 
effect a metamorphosis of the glass from the virgin state to the pulverized state according to the simple linear transformation:

$$
\begin{aligned}
& K(v, D)=K_{0}(v)(1-D)+K_{1}(v) D \\
& G(v, D)=G_{0}(v)(1-D)+G_{1}(v) D \\
& Y(p, D)=Y_{0}(p)(1-D)+Y_{1}(p) D
\end{aligned}
$$

where $G$ is the shear modulus, $K$ is the bulk modulus, and $Y$ is the yield stress, $v$ is the volume, $p$ is the pressure, and $D$ is the damage. The subscripts 0 and 1 refer to virgin and pulverized states respectively. The second term on the right-hand side of the above equations accounts for the observed dilatancy.

\section{Experiments.}

The shaped charges used were $38.1-\mathrm{mm}$ diameter and were manufactured by Physics International, Inc. The liners have a wall thickness of $0.74 \mathrm{~mm}$, a cone-base diameter of $37.7 \mathrm{~mm}$, a cone angle of 42 degrees, and were made of cold drawn, annealed, oxygen-free copper. The explosive mass was $72 \mathrm{~g}$.

The first set of experiments were conducted as follows. An aluminum block, with square cross section, $150 \mathrm{~mm}$ on edge, and $400 \mathrm{~mm}$ long was used as a tamper. Five holes, $35 \mathrm{~mm}$ in diameter, were drilled lengthwise in the tamper. A centered hole was surrounded by the other 4, each placed at the corner of the block. Pyrex rods were then press-fitted in each hole and a $12.5 \mathrm{~mm}$ aluminum cover plate was screwed on the assembly. A shaped charge was then fired at the center hole and penetration was radiographically measured. As expected, the first firing fractured the glass in the other 4 holes, which were then systematically attacked, one-by-one and the penetration was measured after each shot. No significant difference in penetration was evidenced after the first shot (in virgin glass) however, penetration in the shock-damaged material was significantly (about $35 \%$ ) more than in the virgin glass.

To obtain time resolved penetration measurements we used slab targets and instrumented them with printed circuit shorting gages. Each gage consists of two interlocking copper grids supported by a single Kapton substrate. One grid is charged to $+150 \mathrm{~V}$ and the other grid is connected to ground. When the metallic jet pierces a gage the two grids are shorted, thereby discharging a capacitor. The generated signal is recorded digitally by LeCroy recorders and in analogue form by raster scopes. A total of four experiments were performed: Three were against monolithic targets namely aluminum, steel and glass. and one against a composite target made of a glass layer followed by aluminum.

The targets were composed of slabs with a square cross-section of $152.4 \mathrm{~mm}$ on the side and were fastened together with epoxy glue. Figure 2 is a photograph of the experimental setup for the glass target. The standoff distance was 3 charge diameters in all the experiments and was chosen because, at this standoff, the 38.1 -mm precision shaped charge has maximum depth of penetration with minimum scatter. A total of six gages were imbedded in each target. In Figure 3 we plot the penetration as a function of time for the four experiments. The time origin in the figure has been shifted so that zero time corresponds to arrival of the jet tip at the target surface. For the first $20 \mu \mathrm{s}$, the penetrations into glass or aluminum are practically identical. The initial penetration velocity $U$ is about $5 \mathrm{~km} / \mathrm{s}$, in good agreement with hydrodynamic theory for penetration into either target: $\mathrm{U}=\mathrm{V} /[1+$ $\left.\left(\rho_{t} / \rho_{j}\right)^{1 / 2}\right]$, where $V$ is the jet tip velocity $\approx 8 \mathrm{Km} / \mathrm{s}, \rho_{j}$ is the cooper jet density $=8.9 \mathrm{~g} / \mathrm{cm}^{3}$ and $\rho_{t}$ is the target density $=2.7 \mathrm{~g} / \mathrm{cm}^{3}$ for aluminum and $2.3 \mathrm{~g} / \mathrm{cm}^{3}$ for glass.

After about $20 \mu \mathrm{s}$, the data shows a distinct difference between monolithic glass and aluminum, with penetration velocities into the former on the order of one half those in the latter. The low penetration velocity into glass is consistent with measurements by Lawrence and Franz (1986) [6] who obtained a penetration velocity of 2.57 $\mathrm{km} / \mathrm{sec}$ using high-speed framing camera photographs. 
Penetration into the composite target, which had a 78-mm glass layer in front of aluminum, illustrates the effect of the glass on the jet. After penetrating for $20 \mu$ s into the glass, the subsequent penetration into aluminum proceeds at a much slower speed, in fact at a speed comparable to penetration into monolithic glass. Furthermore, the final penetration in the composite target is comparable to the final penetration in monolithic glass. This result demonstrates that the jet is sufficiently degraded, after penetrating only a relatively thin layer of glass, that no further improvement is gained by adding more glass. The composite glass-aluminum target stops the jet in roughly the same thickness as does steel.

\section{Calculations.}

A detailed description of the jet is needed for computer modeling of its penetration. Although this precisionshaped-charge jet tip-velocity is known experimentally to be about $8 \mathrm{~km} / \mathrm{s}$, the diameter of the jet, its length, and velocity gradient have not been measured. We therefore used our computer simulations to obtain those parameters. The jet-formation calculations were performed with LLNL's CALE code [7], a 2D arbitrary Lagrangian-Eulerian hydrocode that is portable to UNIX systems. At a standoff distance of 3 charge diameters, the axial velocity was nearly linear, and the diameter of the jet ranged from $1.5 \mathrm{~mm}$ near the tip to $3 \mathrm{~mm}$ near the slug.

The jet-formation calculation provided the necessary description of the jet which was used in the subsequent penetration calculations. The penetration calculations were run in an Eulerian mode. We used fine zoning, typically $0.25 \mathrm{~mm}$, in the impact region where there is a large pressure gradient. Fine zones were maintained in the impact region throughout the penetration process. This was accomplished by moving the mesh with the speed of the tip of penetrator.

In Figure 4 we show the material boundaries at 5, 10, and $20 \mu$ s from a calculation of jet penetration into glass. The vertical scale in the figure has been expanded to better show the details of the glass-jet interactions. At $5 \mu$ s, a crater is formed around the jet and only the tip of the undisturbed jet interacts with the glass. At $10 \mu \mathrm{s}$, the glass has collapsed on the jet and interacts with it at two locations; and at $20 \mu \mathrm{s}$ it is the disturbed jet that penetrates the target. A jet that is bombarded by glass fragments will break up sooner. The particulated jet is a less effective penetrator for two reasons. First, due to dispersion (wavering), the individual jet particles will not in general impact the target at the same location. The result is a shallower but somewhat larger crater. Second, due to rotation (tumbling) of the individual particles, the projected total length of the jet is shortened; again resulting in a shallower crater. Both dispersion and rotation of the jet particles are stochastic processes.

The qualitative aspects of a stochastic jet-glass interaction are shown in the 2-D plane-strain simulations of Figure 5. In Figure 5a, the glass target had a $1 \%$ higher initial density above the symmetry plane. This slight variation in the target density would have virtually no effect on the final penetration or the shape of the crater in a target material which does not bulk. For a glass target however, the collapse of the glass on the incoming jet (sheet) knocks it out of alignment and reduces the penetration. By comparison, in Figure 5b, we show the jet in a calculation in which symmetry was strictly enforced. Whereas the jet in Figure 5 a which mimmics the 3dimensional world is essentially defeated, the jet in Figure $5 \mathrm{~b}$ continues to penetrate.

Two-dimensional penetration calculations, whether axisymmetric or plane strain, are useful in illustrating the jet interactions with glass but are inherently limited to qualitative descriptions. Three-dimensional calculations are required if rotation and dispersion of the particulated jet are to be quantitatively modeled.

\section{Discussion.}

In this work we have established experimentally that a glass layer is as effective as a monolithic glass target against a shaped charge jet. Further experiments with composite targets can determine the smallest layer of glass needed for the enhanced resistance to penetration. 
An improvement on our predictive capability of jet penetration in glass can be achieved by using a threedimensional hydro-code so that particulated jets can be adequately modeled. DYNA3D [8], JOY [9], MESA3D [10], CTH [11] and ALE3D [12] are codes into which our glass constitutive model can be implemented. Our calculations also suggest that inhomogeneities in certain brittle targets may enhance their performance against jets. Three-dimensional calculations can establish the characteristics of inhomogeneities which are most effective.

A critical parameter in our model is the bulk modulus of the cracked material. This parameter determines the increase in volume of the cracked glass and thus the interaction with the jet. Independent theoretical or experimental estimates of this parameter are highly desirable. A theoretical framework to estimate the reduction of the elastic modulii with increased crack density, using a self-consistent method, has been developed by O'Connell and Budiansky (1974) [13]. The theory is in qualitative agreement with measurements on rocks but has not been tested on glass. Ultrasound measurements of the longitudinal and shear wave speeds can also be used. Rubin and Ahrens (1991) [14] have recently conducted such measurements on various rocks and have indicated the feasability of similar studies on glass [15].

\section{Acknowldegement}

This work was performed under the auspices of the U.S. Department of Energy by Lawrence Livermore National Laboratory under contract No. W-7405-Eng 48

\section{References.}

1. Eichelberger, R. J., 1966. Protection against shaped charge warhead, Proceedings of the second symposium on lightweight armor materials, volume II, Mellon Institute, Pittsburgh, PA, 5-6 May.

2. Solve, G. and Cagnoux, J. 1989. The behavior of Pyrex glass against a shaped-charge jet, Proceedings of the American Physical Society topical conference on shock waves in condensed matter, Albuquerque, NM, 14-17 August.

3. Glenn, L.A., Moran, B. and Kusubov, A. S. Modeling jet penetration in glass, Proceedings of the conference on the Application of 3-D Hydrocodes to Armor/Anti-Armor Problems BRL, Aberdeen Proving Grounds, MD, 8-9 May 1990

4. Glenn, L. A., 1976. The fracture of a glass half-space by projectile impact, J. Mech. Phys. Solids, 24, 93-106.

5. Moran, B., Glenn, L. A., and Kusubov, A., Experiments and calculations of jet penetration in glass, Proceedings of the Combat Vehicle Survivability Symposium, Gaithersburg, Maryland, April 14-17, 1991.

6. Lawrence, W. and Franz, R. E. Stress measurements in glass using shaped-charge jets. Proceedings of the Hypervelocity Impact Symposium at San Antonio, Texas, 21-24 October 1986.

7. Tipton, R. CALE User's Manual, University of California Lawrence Livermore National Laboratory (1990).

8. Hallquist, J.O. User's Manual for DYNA3D and DYNAP (Nonlinear Dynamic Analysis of Solids in Three Dimensions), University of California, Lawrence Livermore National Laboratory Rept. UCID-19156 (1981).

9. Couch, R., Albright, E., Alexander, N. The JOY Computer Code. Lawrence Livermore National Laboratory Report UCID 19688, January 1983.

10. Cagliostro, D. J., Mandell, D. A. , Schwalbe, L. A., Adams, T. F., and Chapyak, E. J, MESA3D calculations: Armor penetration by projectile with combined obliquity and yaw. International Journal of Impact Engineering Vol 10, p 81-92 (1990). 
11. McGlaun, M. J., Thompson, S. L., Kmetyk, L. N., and Elrick, M. J., A brief description of the 3 dimensional shock wave physics code CTH. Sandia National Laboratory Report. SAND89-0607, July 1990.

12. Sharpe, R. ALE3D under development, Private communications April, 1991.

13. O'Connell, R. J. and Budiansky, B. 1974. Seismic velocities in dry and saturated cracked solids, I. Geophys. Res. $79.5412-5426$.

14. Rubin, A. M. and Ahrens, T. J. Dynamic tensile failure induced velocity deficits in rock., Geophys. Res. Lett. Vol 18 pp 219-222, Feb 1991

15. (Private communication, March 1991)

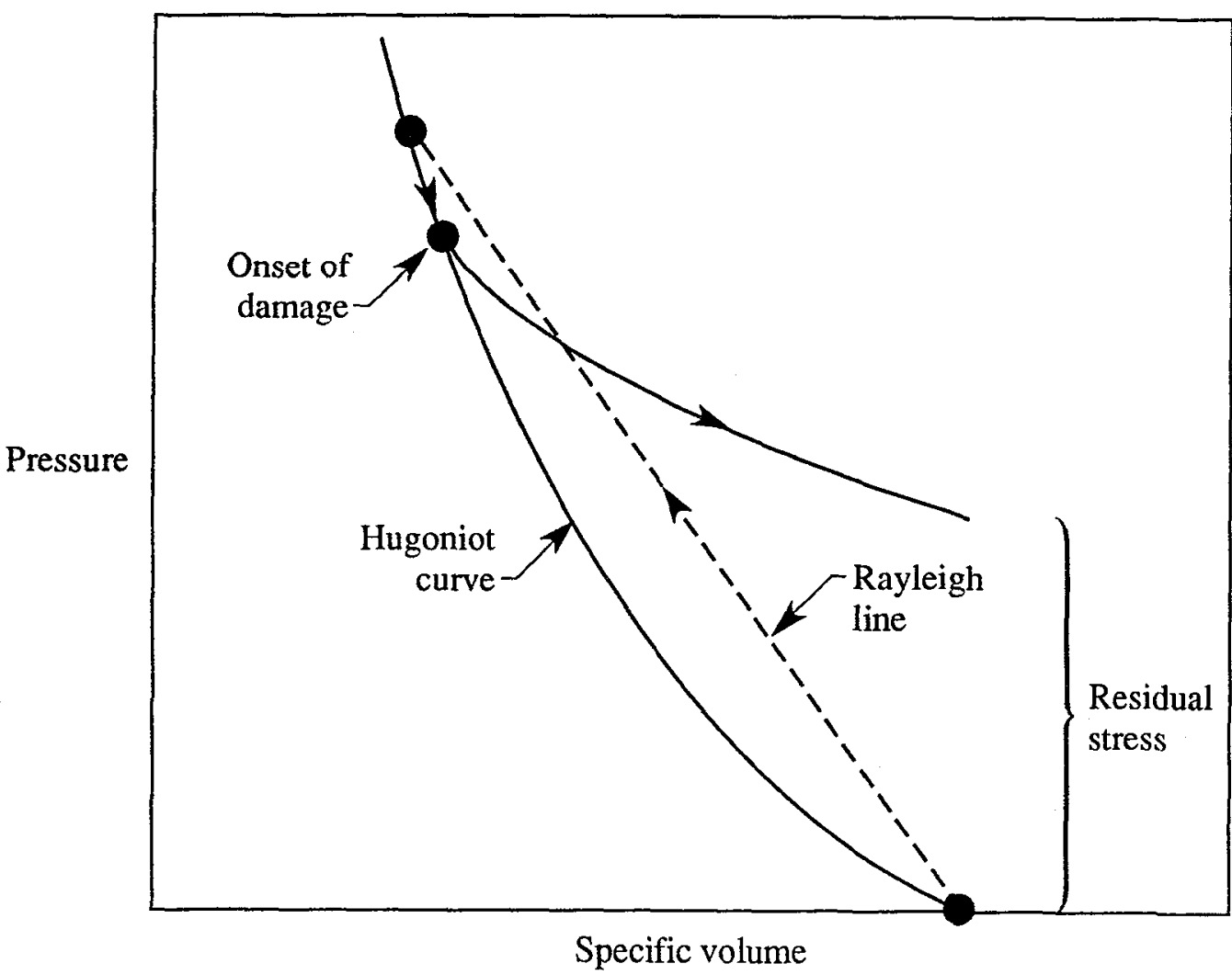

Figure 1: Constitutive model for glass showing shock loading and unloading 


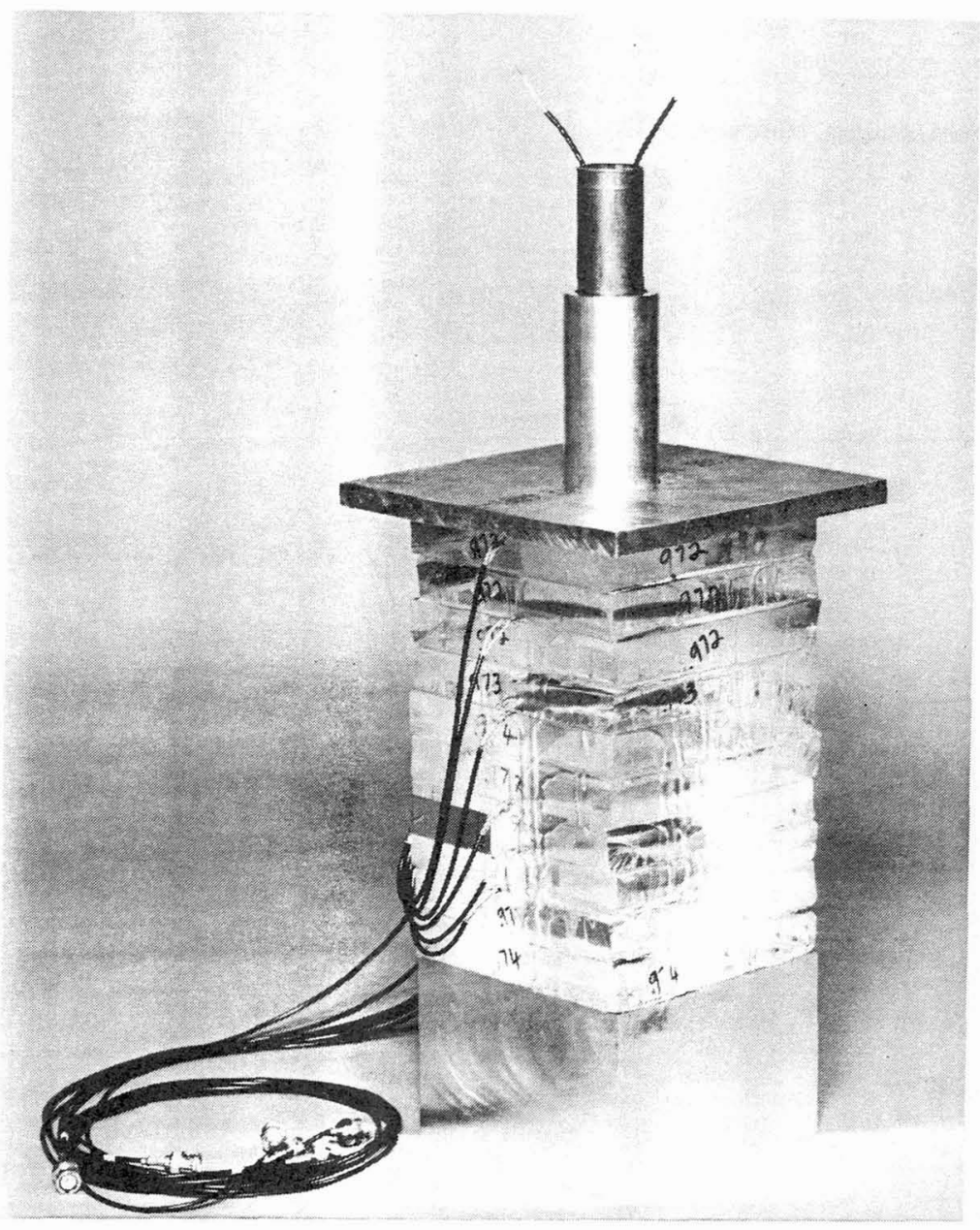

Figure 2: Photograph of the experimental setup for the glass target. 


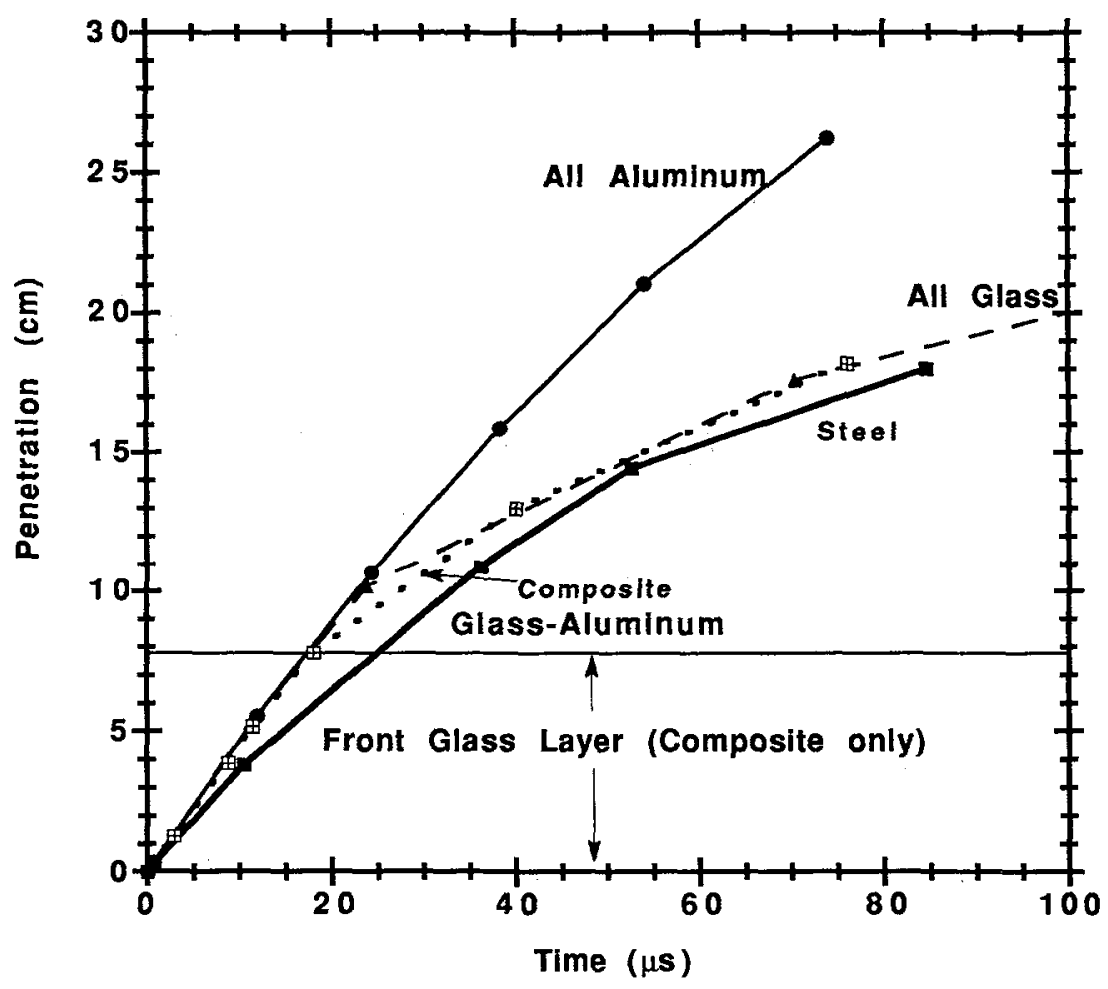

Figure 3: Penetration as a function of time for several targets 

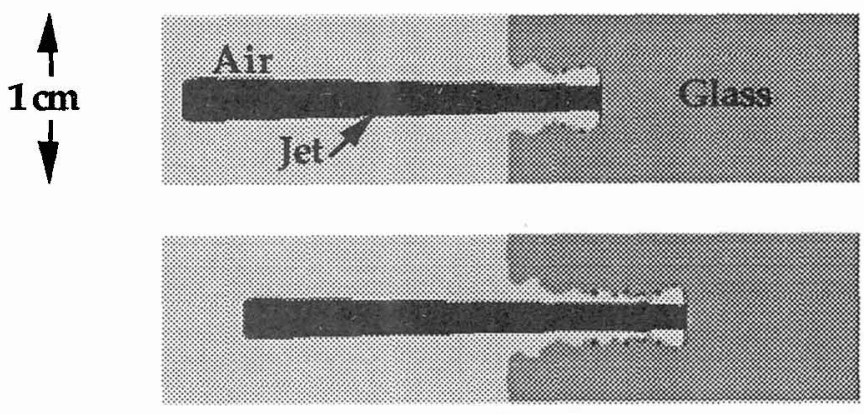

$10 \mu \mathrm{s}$

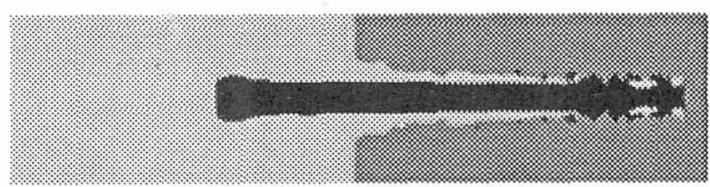

$20 \mu \mathrm{s}$ $18 \mathrm{~cm}$

Figure 4: Calculated material boundaries at 5, 10, and $20 \mathrm{~ms}$. Glass fragments interact with the jet after formation of an initial crater.
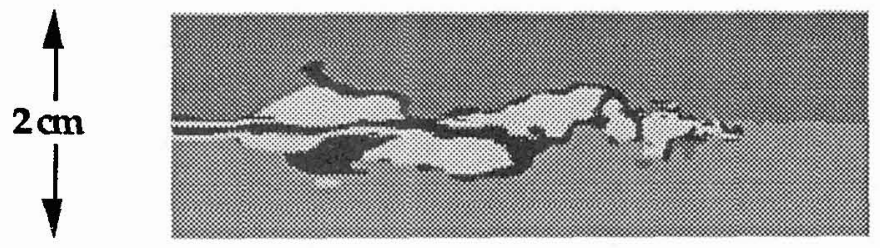

(a)

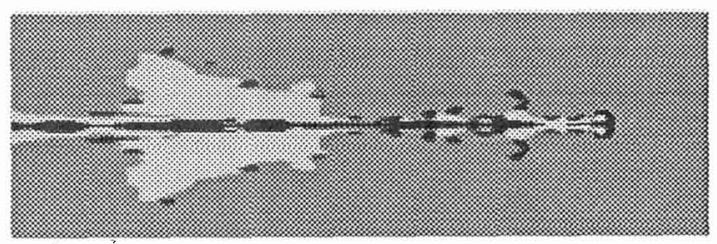

(b)

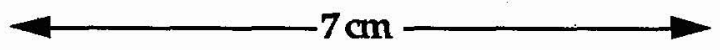

Figure 5: Plane strain simulations of a jet into glass. The unaligned jet in (a) is an ineffective jet. 